\title{
39. DETERMINATION OF PLANETARY MASSES FROM THE MOTIONS OF COMETS
}

\author{
W. J. KLEPCZYNSKI \\ U.S. Naval Observatory, Washington, D.C., U.S.A.
}

\begin{abstract}
A brief survey is given of past determinations of the masses of the principal planets from analyses of the motions of comets. Some numerical experiments using comets which have close approaches to Jupiter are made. As a result of these experiments, it is concluded that the conventional least squares solution for the correction to the mass of Jupiter is inadequate for comets which have a close approach to Jupiter. It is further concluded that perhaps, in some cases, the apparent presence of nongravitational forces is merely a manifestation of the failure of the conventional orbit correction process to adjust correctly the orbits of objects which undergo very large perturbations, and it also may be a consequence of errors in the adopted planetary masses. It is suggested that the use of partial derivatives obtained through the numerical integration of the variational equations may overcome the difficulties.
\end{abstract}

\section{Introduction}

The determination of the mass of a planet is based on (1) an analysis of the motion of a satellite, if it possesses one; (2) an analysis of the secular or periodic perturbations it produces in the motion of another celestial object or; (3) an analysis of the large perturbations induced in the trajectory of an object during an especially close approach to the body whose mass is being sought.

The last case offers many advantages over the other methods, the primary ones being that the observational history of the object whose motion is being studied does not have to be as long as in the other methods, and that the magnitude of this type of perturbation is considerably larger than in the second method.

Comets should be extremely useful objects for determining the masses of the planets because they can come closer to the major planets than any other class of celestial objects. In the case of Jupiter, close approaches by comets are relatively common occurrences, and a careful analysis of their motions would be very useful in improving the knowledge of the mass of this great planet. However, this has not been the case. There have not been very many determinations of planetary masses based on analyses of the motions of comets, and those that have been made give widely varying results.

This investigation was undertaken with the hope of finding a plausible explanation for the disparate results among the various investigations and to suggest possible avenues for future investigations.

\section{Mass Determinations from Cometary Motion}

Since the late nineteenth century there have been fourteen investigations which have attempted to determine the mass of a planet through perturbations induced in the 
TABLE I

Planetary mass determinations based on analysis of cometary motion

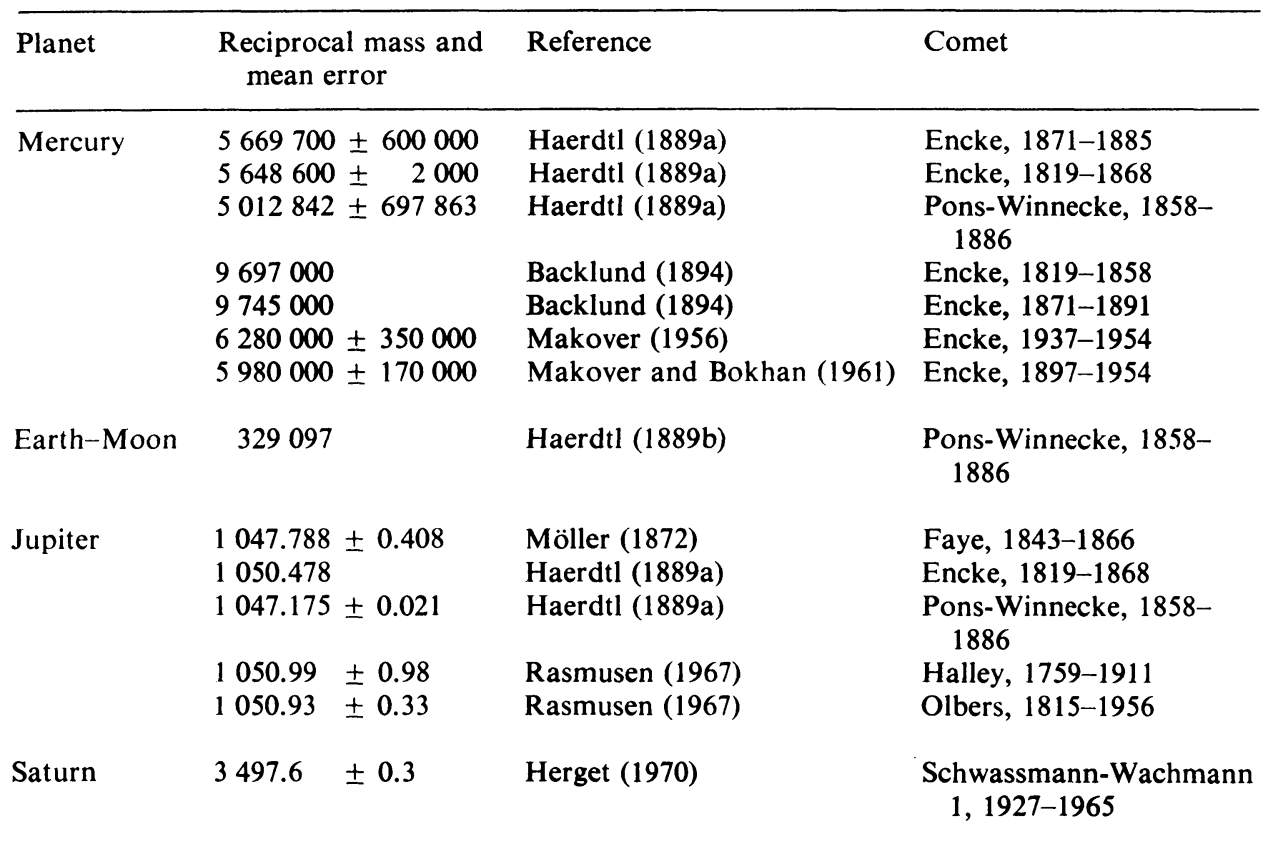

motions of comets; see Table I. The determinations primarily analyze large perturbations produced in the motion of the comet caused by a close approach to the planet. An inspection of Table I shows that the results of these investigations are not in agreement with the values determined using other techniques and objects, except for the investigations of Herget (1970) and of Makover and Bokhan (1961).

Attempts have been made to attribute these discrepancies and other deficiencies noted in studies of the motions of comets to errors of observation, inaccurate numerical integrations of the orbits, nongravitational forces, or other unknown cause. True, there are many observational problems associated with the determination of the position of a comet. However, in the case of a close approach to a planet, the effects we are looking for should be an order of magnitude greater than the errors of observation. Furthermore, current procedures of astrometry have greatly reduced the errors associated with cometary positions. The use of electronic computers has all but eliminated the problems commonly associated with the numerical integrations of orbits. The existence of nongravitational forces is generally accepted (Marsden, 1968, 1969, 1970); however, their magnitude and effect on the observed motions of comets have been questioned (Roemer, 1961).

There are two error sources which, I believe, may affect the results of analyses made thus far: first, the system of planetary masses used in the investigations may be in error; second, the approximations used in the differential correction process may not be sufficiently accurate. 


\section{Comments on Adopted Masses and the Orbit Correction Process}

It is an accepted fact that the currently adopted IAU set of planetary masses is not necessarily the best set. Roemer (1961) has pointed out that this might explain some of the irregularities observed in the motions of comets. Since we normally use the technique of successive approximations in our analysis of the motion of celestial objects, inaccurate initial conditions in our numerical integrations and in the differential correction process can drastically affect our results. Marsden (1972) achieved a significant improvement in the orbit of the minor planet 944 Hidalgo by including in his analysis a solution for a correction to the mass of Saturn.

The equations we use to make a differential correction of the initial values of the osculating orbital elements and other constants which affect the motion of a celestial object are also subject to some approximations. The partial derivatives which form the coefficients of the unknowns used in our equations of condition can be formed in several ways. Numerically integrating the variational equations is considered the best method to use in order to obtain accurate partial derivatives. In fact, this is the procedure used by Herget (1970) in his study of the motion of P/Schwassmann-Wachmann 1. Another method of obtaining accurate partial derivatives makes use of a general theory of the motion of a comet. In this case, the only limitation on the accuracy of the partial derivatives is the accuracy of the general theory.

The two approaches just mentioned require extensive computation, which is not practical for a large number of investigators. As a result other methods have been introduced to obtain the partial derivatives. The technique of Eckert and Brouwer (1937) is one approximate method for evaluating the partial derivatives which has gained wide acceptance. Its application implicitly assumes that the real orbit of the object does not differ significantly from the osculating orbit. Another technique frequently used to evaluate a partial derivative is to difference two numerical integrations which use two different values for the unknown in question and identical values for all other parameters. The major drawback of this method is that we do not know for how large a difference of the variable in question this procedure is valid.

In this investigation use is made of the last two techniques: the partial derivatives for the coefficients of the corrections to the orbital elements are obtained using the method of Eckert and Brouwer (1937), while the partial derivative for the coefficient of the correction to the disturbing mass is obtained by differencing two numerical integrations which utilize the same osculating elements but two different values for the mass of Jupiter.

\section{Numerical Experiments}

In order to determine the effect of an error in the adopted mass of a disturbing body and to test the adequacy of the differential correction procedure just described, the following numerical experiment was performed.

Six comets were selected which had close approaches to Jupiter. They were $\mathrm{P} /$ Brooks 2, P/Grigg-Skjellerup, P/Kopff, P/Oterma, P/Pons-Winnecke, and P/Wolf. Elements 
for these six comets were given by Porter (1961). The dates of perihelion passage were rounded to the nearest half day for computational convenience. Therefore, the elements used in this study are only a reasonable approximation to those of the real objects. For this reason, Table II lists only approximate osculating elements of these objects for the epoch of the integration, JED 2434000.5.

TABLE II

Approximate osculating elements of comets used in this investigation (epoch JED 2434000.5)

\begin{tabular}{lllcccc}
\hline & Brooks 2 & Grigg-Skjellerup & Kopff & Oterma & Pons-Winnecke & Wolf \\
\hline$l_{0}$ & $275^{\circ}$ & $344^{\circ}$ & $10^{\circ}$ & $65^{\circ}$ & $16^{\circ}$ & $49^{\circ}$ \\
$\omega$ & $196^{\circ}$ & $356^{\circ}$ & $32^{\circ}$ & $355^{\circ}$ & $170^{\circ}$ & $161^{\circ}$ \\
$\Omega$ & $178^{\circ}$ & $215^{\circ}$ & $253^{\circ}$ & $155^{\circ}$ & $94^{\circ}$ & $204^{\circ}$ \\
$i$ & $6^{\circ}$ & $18^{\circ}$ & $7^{\circ}$ & $4^{\circ}$ & $22^{\circ}$ & $27^{\circ}$ \\
$e$ & 0.487 & 0.704 & 0.556 & 0.143 & 0.653 & 0.396 \\
$a$ & 3.638 & 2.887 & 3.369 & 3.971 & 3.347 & 4.134 \\
$n$ & 511 & 723 & 574 & 448 & 579 & 422 \\
$P$ & 6.938 & 4.905 & 6.183 & 7.912 & 6.124 & 8.405 \\
$q$ & 1.867 & 0.856 & 1.495 & 3.404 & 1.161 & 2.498 \\
$Q$ & 5.408 & 4.918 & 5.242 & 4.538 & 5.534 & 5.770 \\
\hline
\end{tabular}

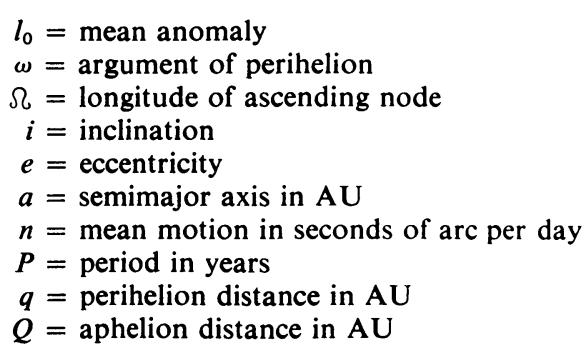

Using the numerical integration program by Schubart and Stumpff (1966), a simultaneous integration of the equations of motion of the planets Venus through Pluto and the six comets was performed at a half-day interval, backward from the epoch of integration for a period of $60 \mathrm{yr}$. This integration used the IAU values for the planetary masses, the reciprocal mass of Jupiter in this set being 1047.355. The heliocentric rectangular coordinates which resulted from this integration were transformed into heliocentric longitude and latitude. This set of coordinates became the standard of comparison for all subsequent studies and is referred to as our standard set of observations.

In order to see the character of the orbits of the six comets used in this investigation, the paths of these objects were plotted in a rotating frame of reference with Jupiter fixed on the abscissa at unit distance and the frame rotating with the same rate as Jupiter (Figure 1). The comet with the minimum distance from Jupiter at the time of closest approach is $\mathrm{P} /$ Brooks 2 . In increasing minimum distance we next have $\mathrm{P} / \mathrm{Wolf}$, followed by $\mathrm{P} /$ Oterma, $\mathrm{P} /$ Grigg-Skjellerup, $\mathrm{P} / \mathrm{Pons}$-Winnecke, and finally $\mathrm{P} / \mathrm{Kopff}$. 
COMET P/BROOKS 2

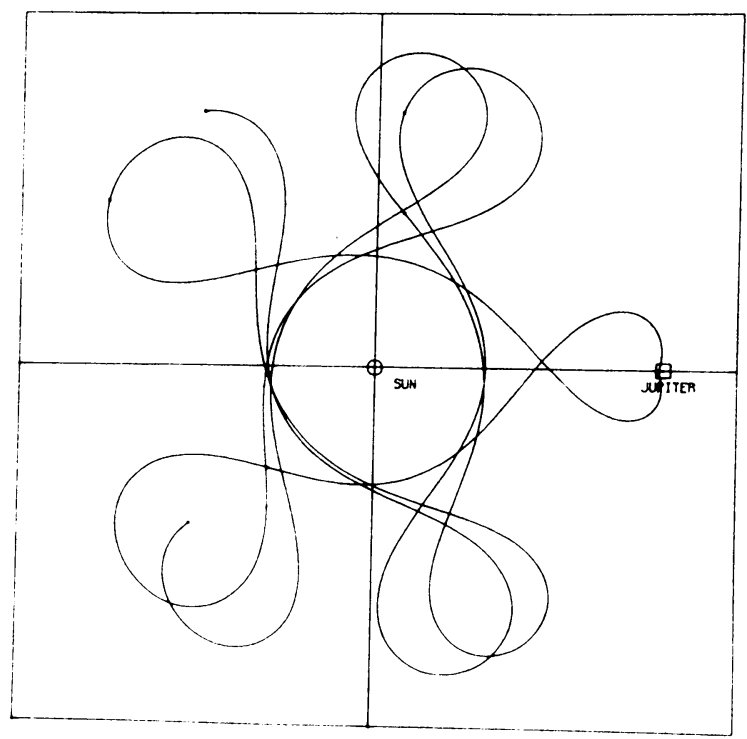

Fig. $1 \mathrm{a}$.

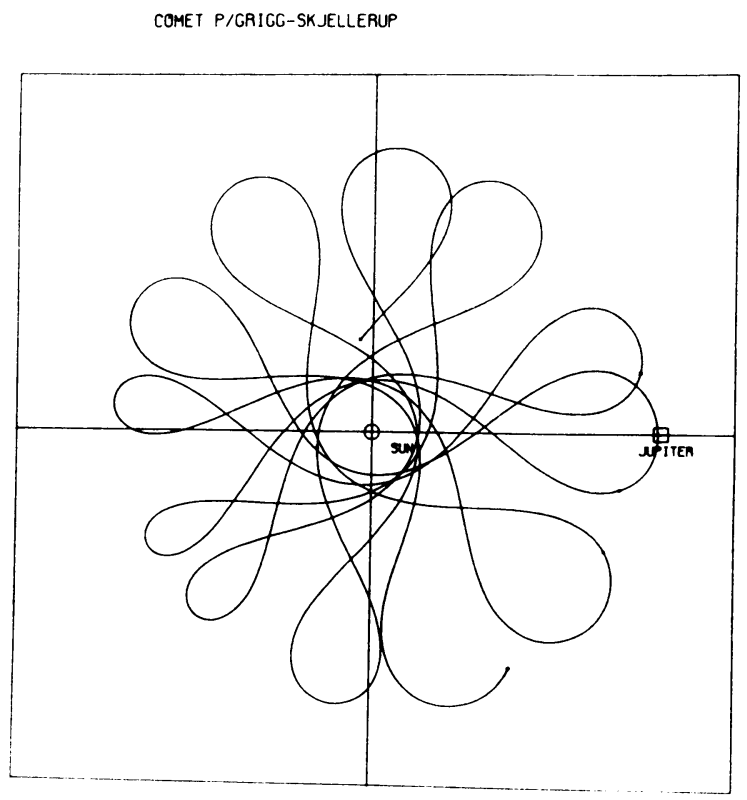

Fig. 1b. 
COMET P/KOPFF

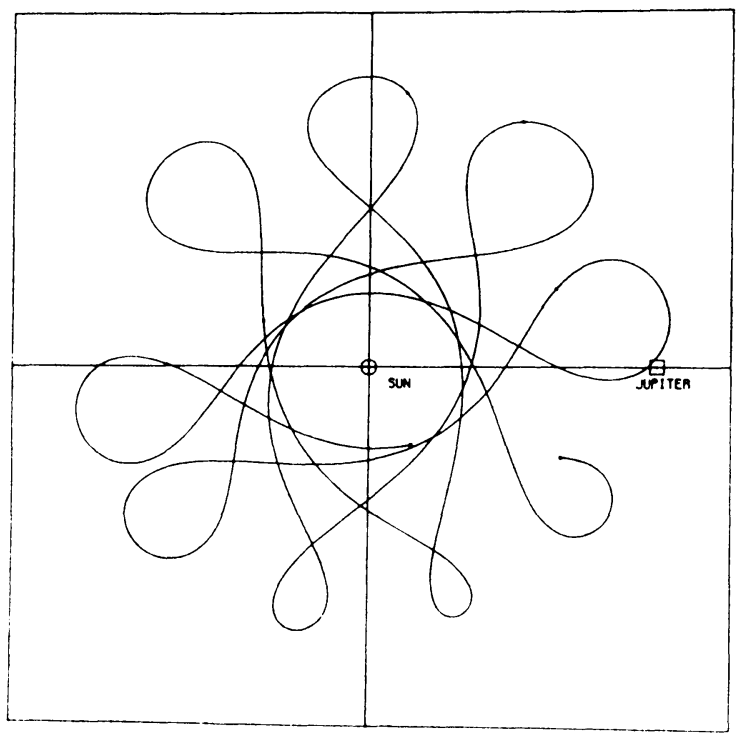

Fig. 1c.

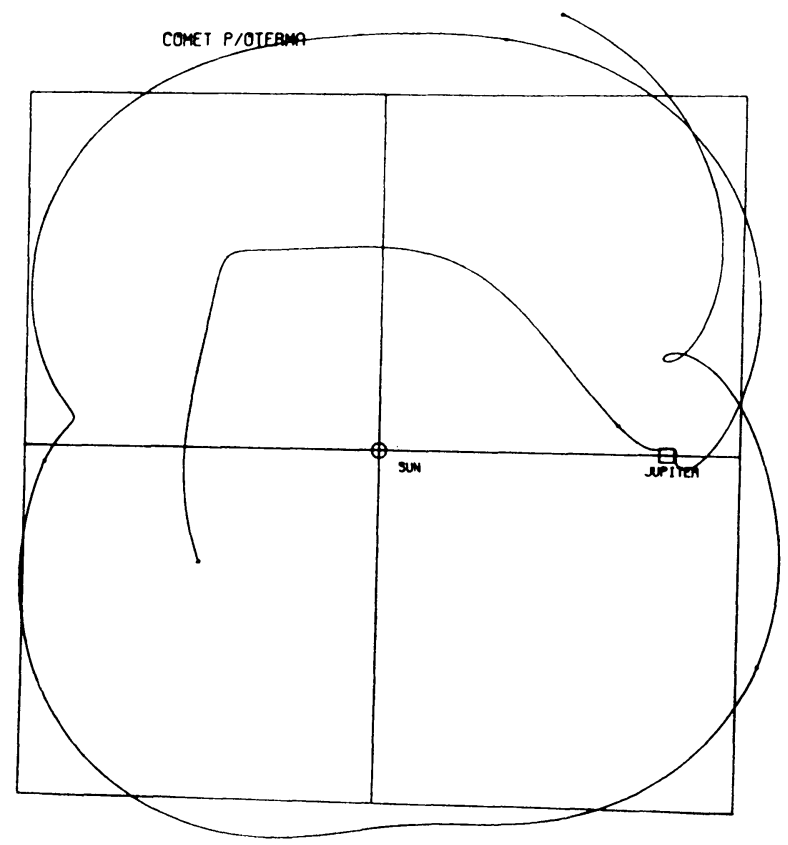

Fig. 1d. 
CONET P/PONS-HINNECKE

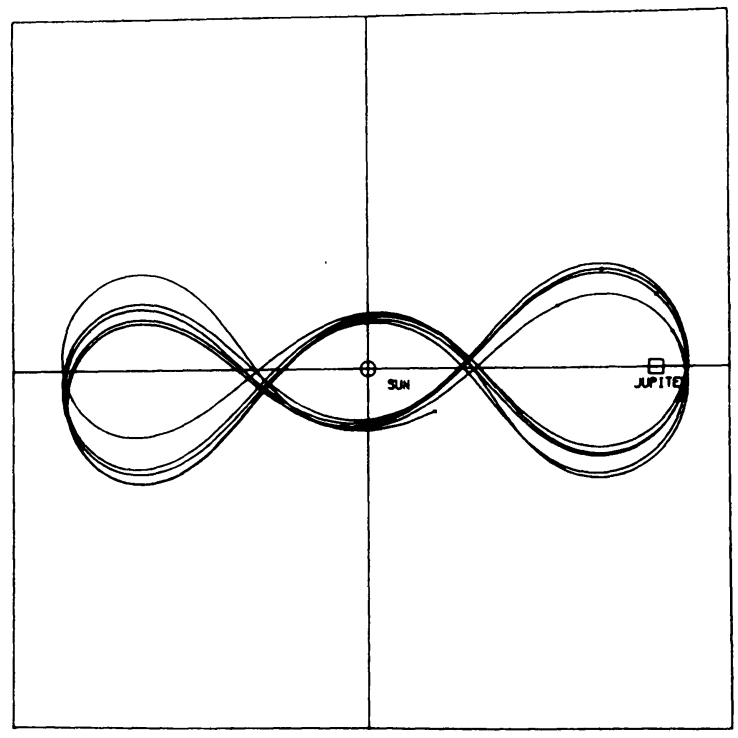

Fig. le.

COMET P/HOLF

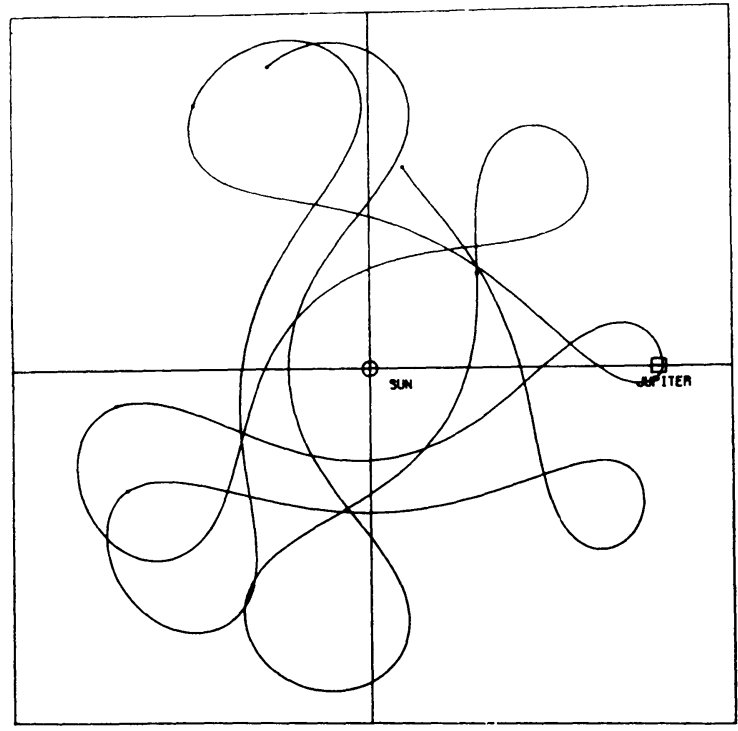

Fig. 1f.

Figs. 1a-f. Orbits of comets used in this investigation plotted in rotating frame of reference. Jupiter is at unit distance and the frame is rotating with the actual velocity of Jupiter. 
Next, keeping all other parameters fixed, the disturbing mass of Jupiter was changed to 1047.390 , and a new integration was performed. This new integration is sometimes referred to as the 'unfitted' integration. This new value of Jupiter's mass corresponds to a change of 0.035 or $0.003 \%$ in the reciprocal mass. The differences in heliocentric longitude for each comet between this integration and the standard integration which used 1047.355 as the reciprocal mass of Jupiter are exhibited in Figure 2. Note that there is one vertical line corresponding to the epoch of integration and one at the point of closest approach of each comet to Jupiter.

An attempt was then made to fit the integration which utilized 1047.390 as the disturbing mass of Jupiter to our standard artificial observations. Two classes of solutions were made. In the first class of solutions, the observational subset which went into the differential correction program included those observations for each comet from the epoch of the integration to some date beyond the point of closest approach. In the second class of solution, the differential correction was based on the entire span of our standard artificial observations. In both classes, new integrations based on the new elements were made.

In both classes, after new integrations had been formed, two types of solutions were made: one involved corrections to the six orbital elements; the other added a seventh unknown to correct the mass of Jupiter. The six-unknown solution was made to test the adequacy of the previous correction. The coefficient of the six unknowns, the partial derivatives, were determined by the method of Eckert and Brouwer. The unknowns actually used are those designated Set III in Brouwer and Clemence (1961).

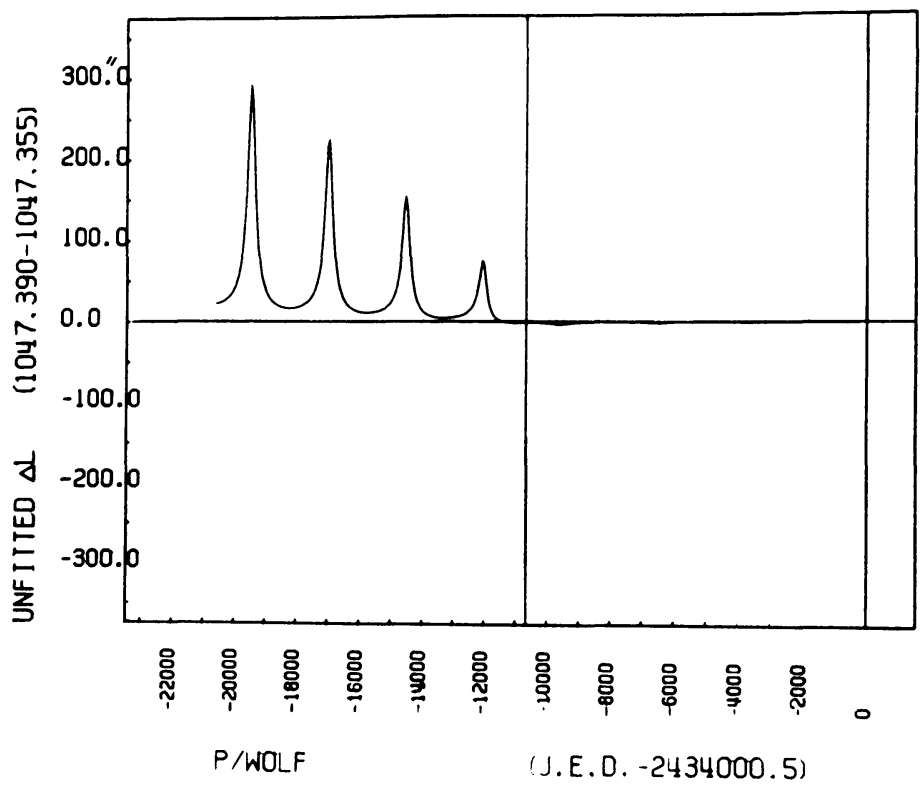

Fig. 2a. 


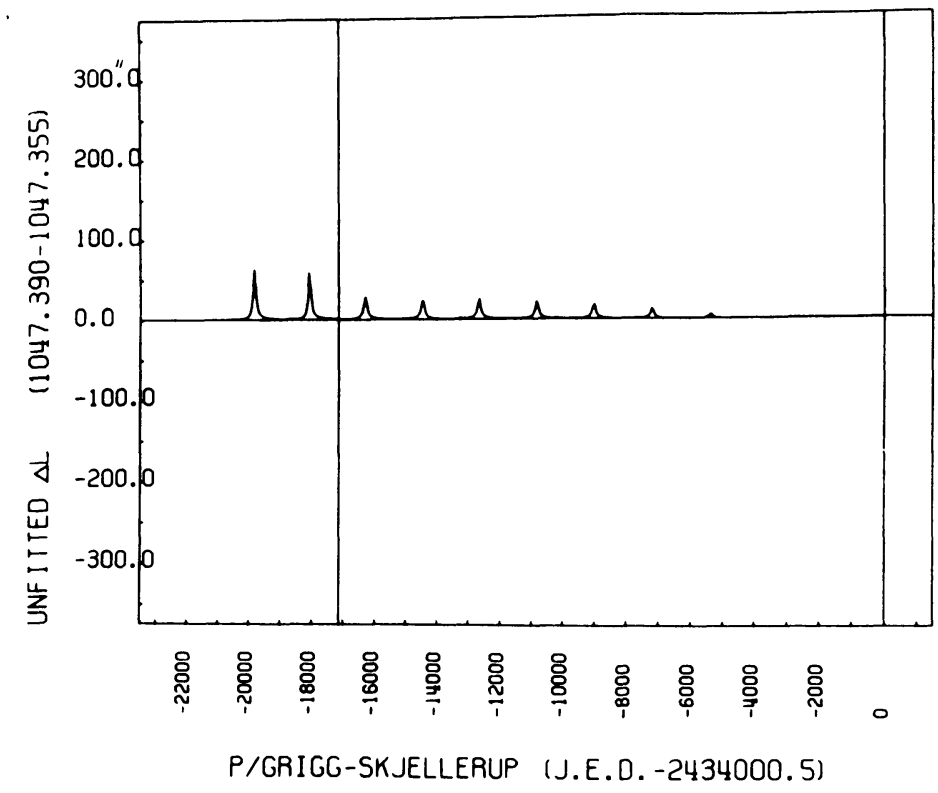

Fig. 2b.

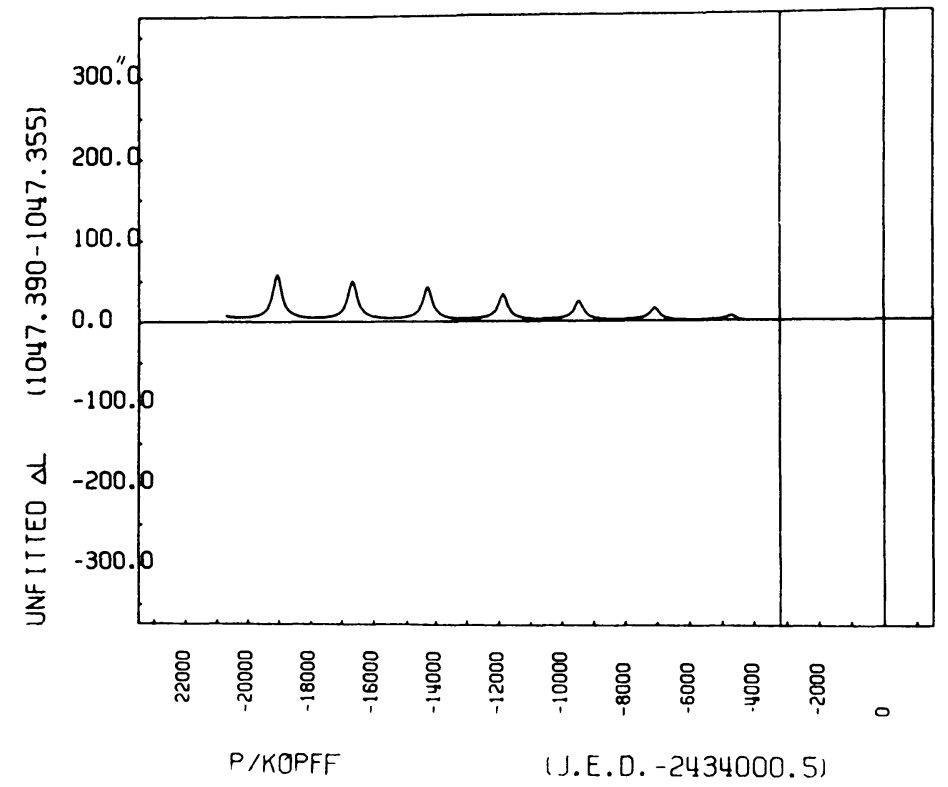

Fig. 2c. 


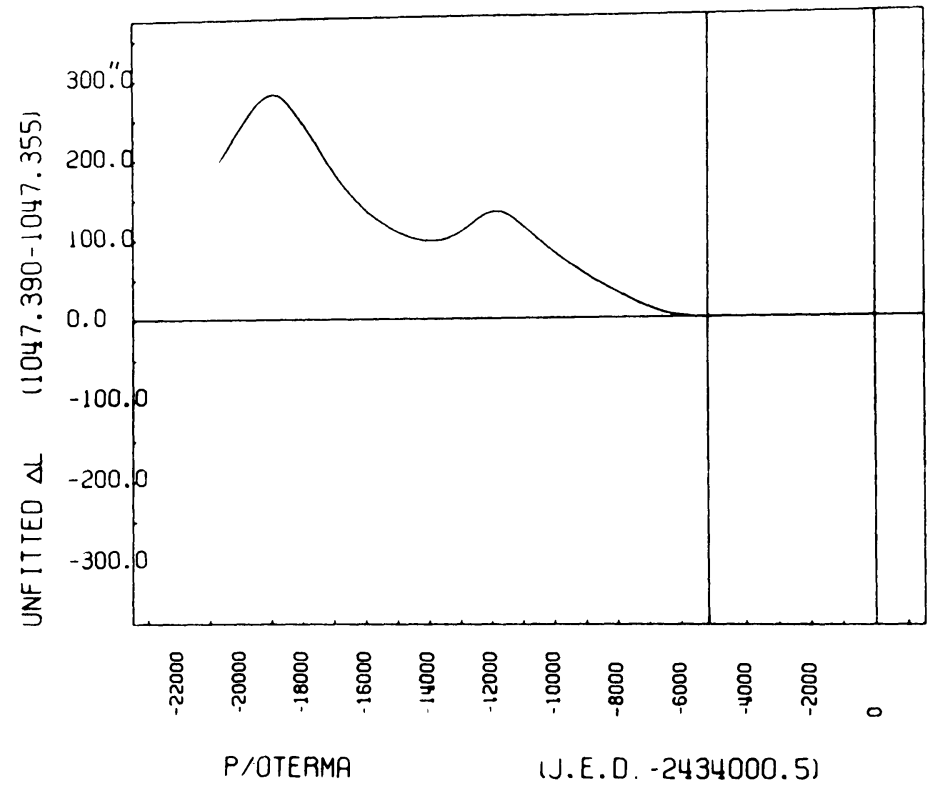

Fig. 2d.

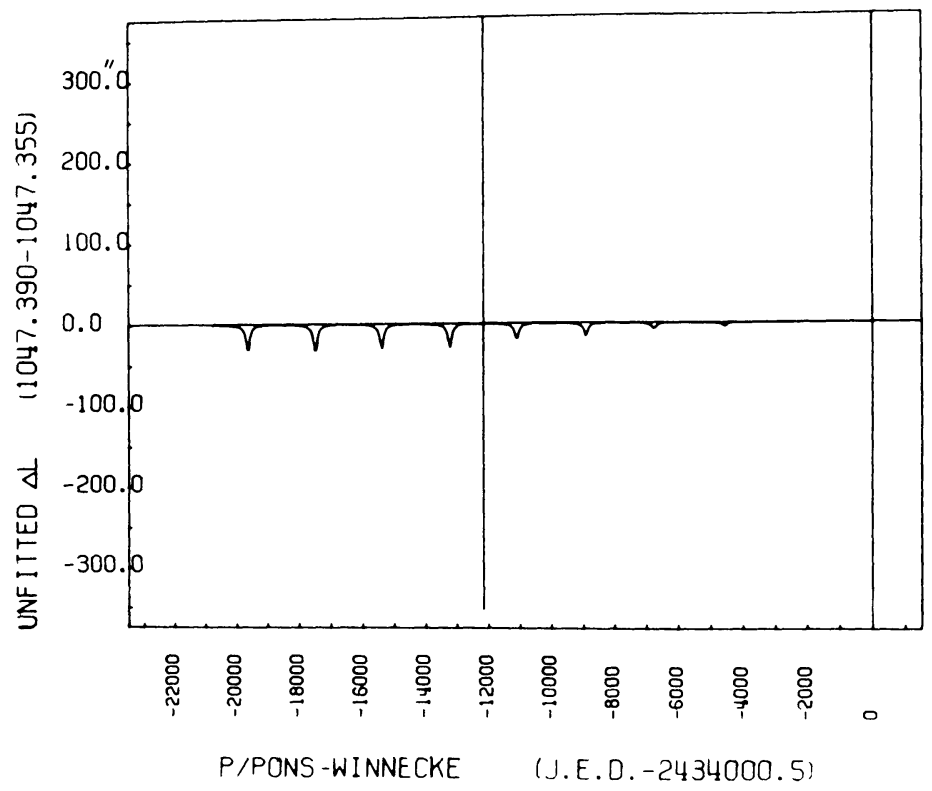

Fig. 2e. 


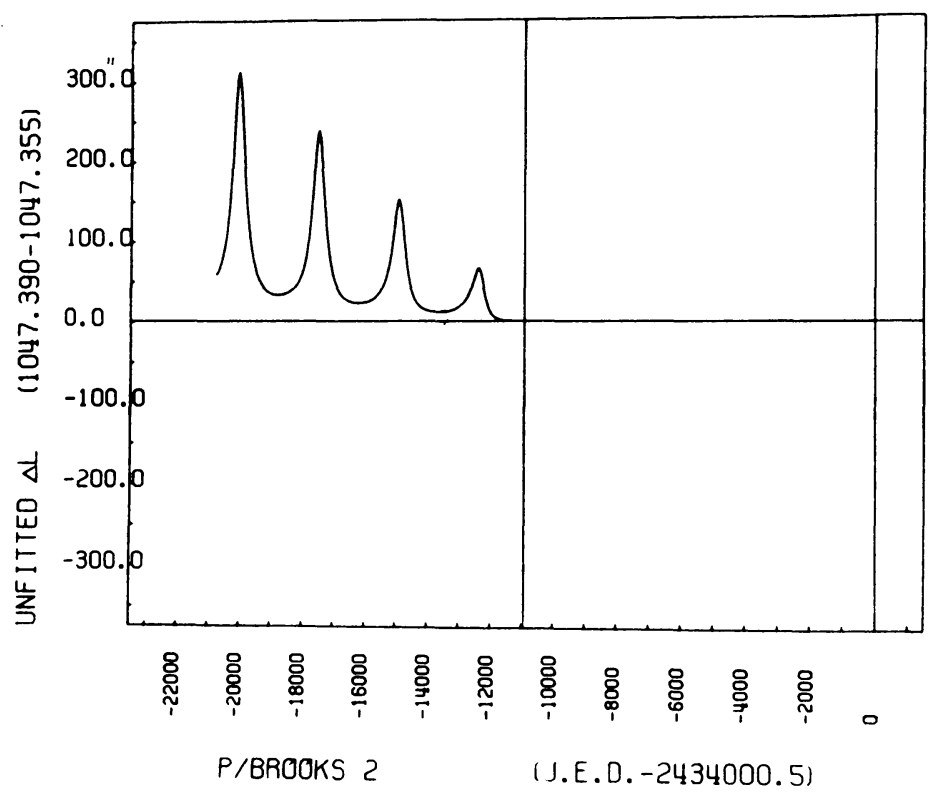

Fig. 2f.

Figs. 2a-f. For the six comets used in this investigation, the differences in heliocentric longitude between the standard numerical integration and one which uses the same osculating elements for each object but 1047.390 for the reciprocal mass of Jupiter are given in seconds of arc.

The coefficient of the seventh unknown in the equations of condition was formed by the differencing techniques already described.

\section{Results of Numerical Experiments}

The attempts to fit an integration based on 1047.390 for the mass of Jupiter to the standard observations extending from the epoch of the integration to beyond the points of closest approach were extremely successful. Figure 3 is a plot of the differences in orbital longitude for each of the comets between the standard observations and the integration fitted to the close approach data. Since several close approaches of $\mathbf{P} /$ Pons-Winnecke had inadvertently been included in the fitting process, not one as had been desired, the results for this comet are not similar to those of the others, and it is omitted from this part of the discussion.

After fitting the close approach observations with the six-unknown solutions, a seven-unknown solution was made. For each comet the resulting value for the correction to the mass of Jupiter should be -0.035 , yielding a value of 1047.355 for the true mass of Jupiter. The results contained in Table III are listed in a chronological order where time is measured in days after close approach; that is to say, the observations which were used in the differential correction process included close approach plus 


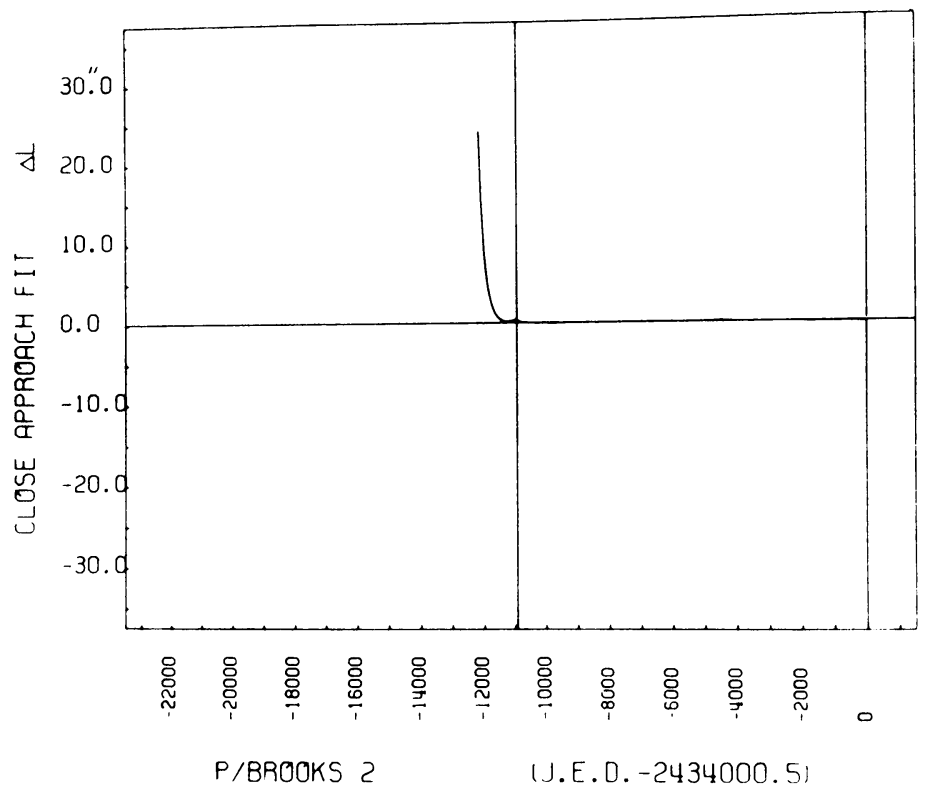

Fig. 3a.

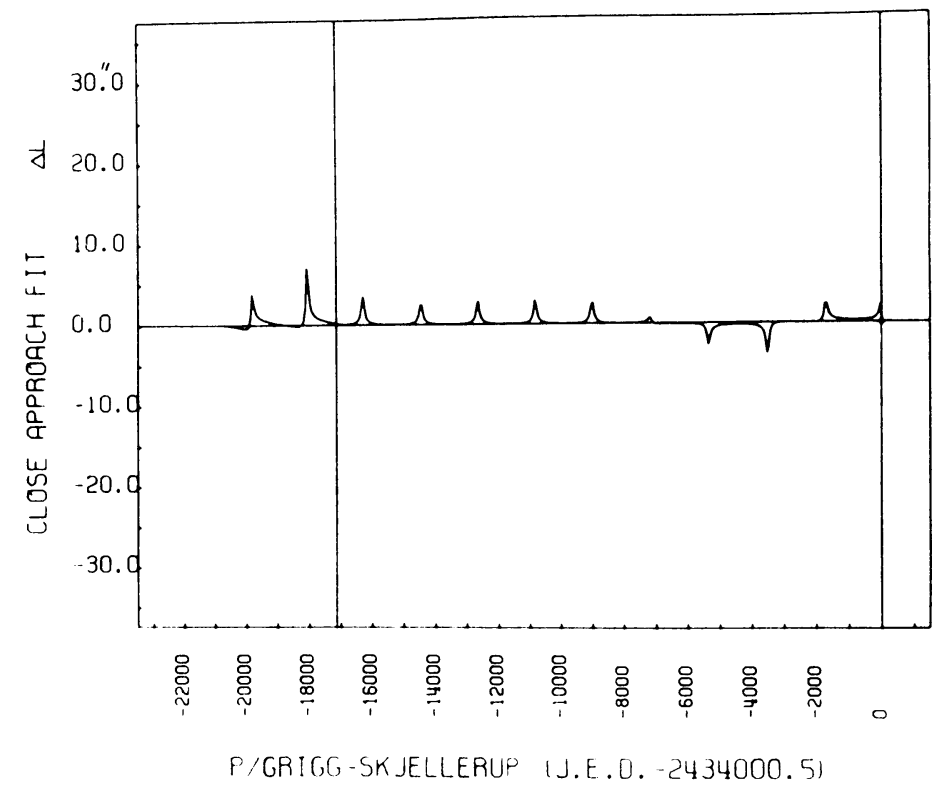

Fig. 3b. 


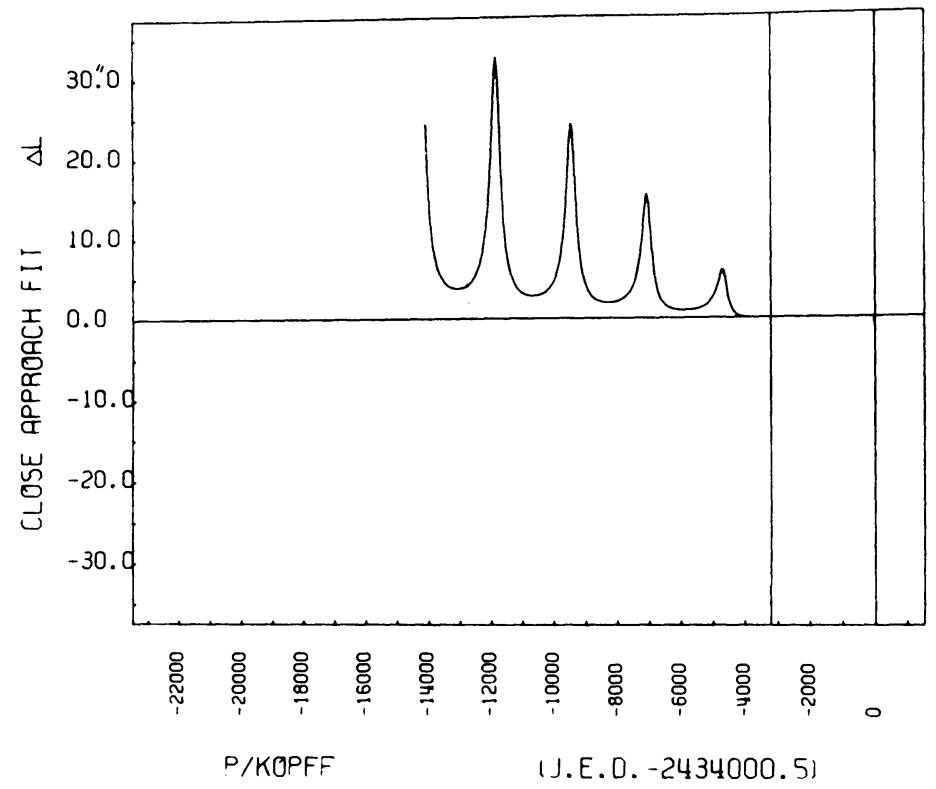

Fig. 3c.

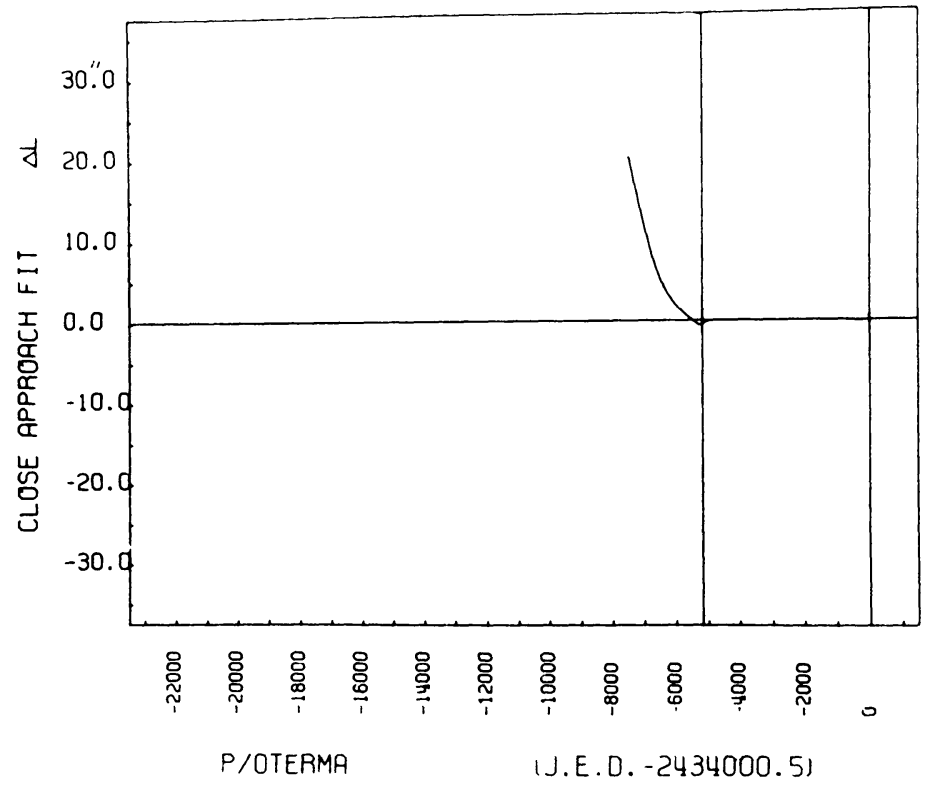

Fig. 3d. 


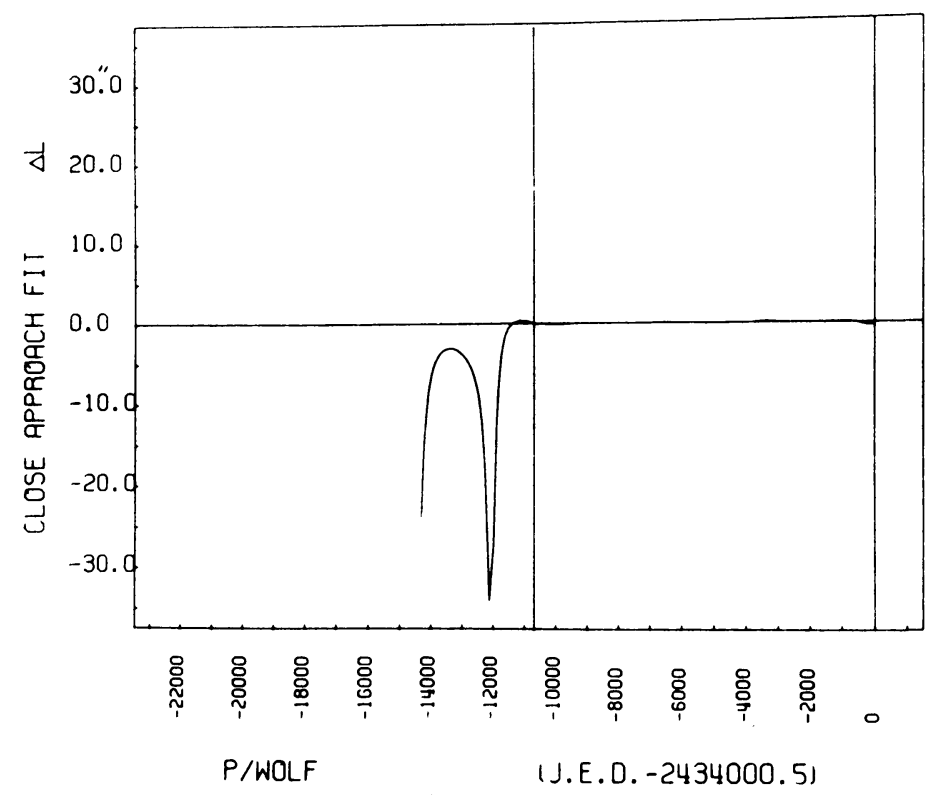

Fig. 3e.

Figs. 3a-e. For five comets used in this investigation, the difference in heliocentric longitude between the standard numerical integration and a numerical integration, which uses 1047.390 for the reciprocal mass of Jupiter and which has been fitted to the close approach observational set, are given in seconds of arc.

TABLE III

Solution for reciprocal mass of Jupiter based on close approach data set

\begin{tabular}{lrlll}
\hline Comet & \multicolumn{1}{l}{$t$} & $\Delta m$ & $\rho$ & \multicolumn{1}{l}{$T$} \\
\hline P/Wolf & 25 & -0.035 & 0.119 & 2423325 \\
P/Brooks 2 & 65 & -0.034 & 0.089 & 2423065 \\
P/Kopf & 93 & -0.036 & 0.577 & 2430793 \\
P/Oterma & 126 & -0.030 & 0.166 & 2428826 \\
P/Grigg-Skjellerup & 167 & -0.018 & 0.173 & 2416867 \\
\hline
\end{tabular}

$\begin{aligned} t & =\text { number of days after close approach } \\ \Delta m & =\text { correction to reciprocal mass of Jupiter } \\ \rho & =\text { distance of minimum separation } \\ T & =\text { approximate JED of close approach }\end{aligned}$

the indicated number of days beyond. Also included in the table is the distance of minimum separation.

The attempts to fit an integration using 1047.390 as the assumed mass of Jupiter to the entire period covered by the standard observations were not very successful. It was only possible to fit three of the comet orbits to the observations. These three 


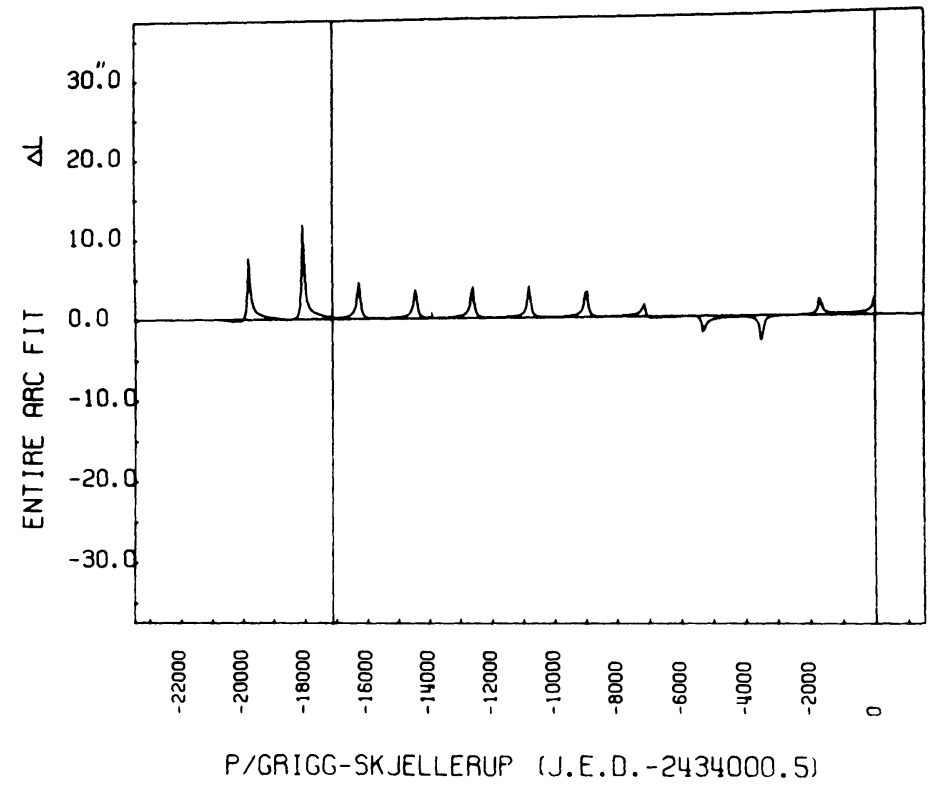

Fig. 4a.

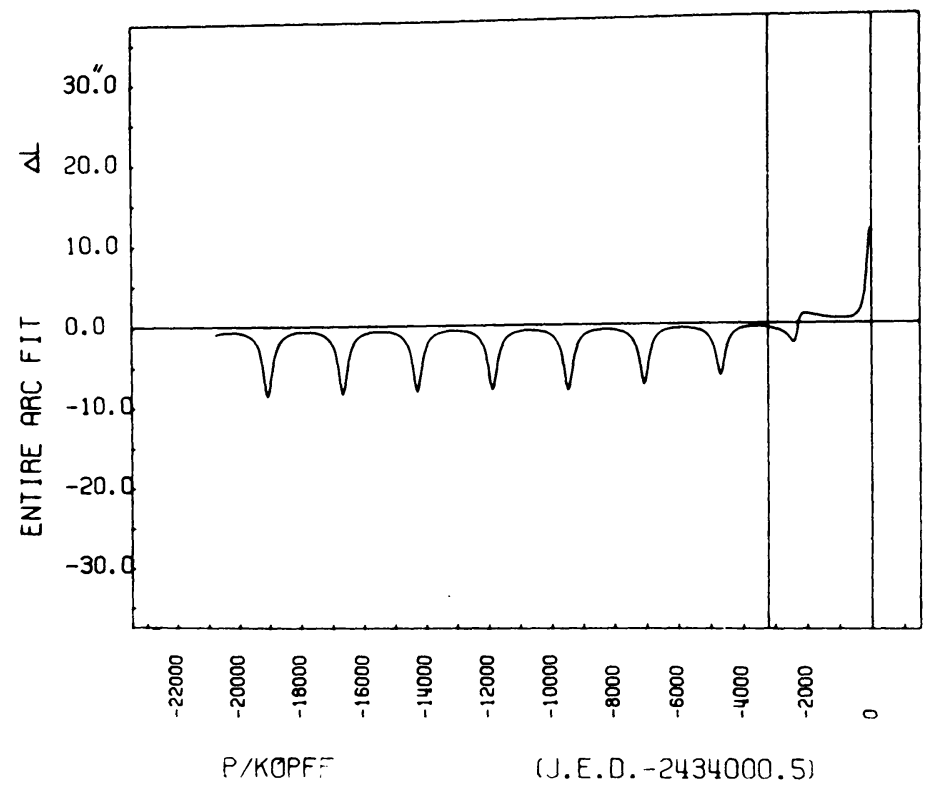

Fig. 4b. 


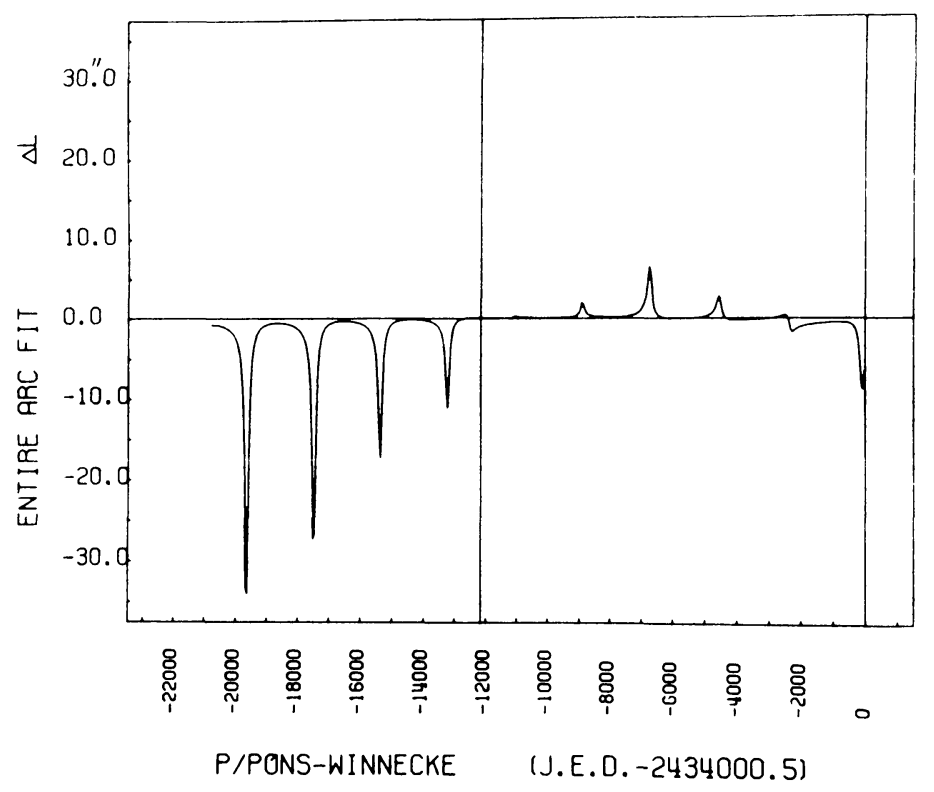

Fig. 4c.

Figs. 4a-c. For three comets used in this investigation, the differences in heliocentric longitude between the standard numerical integration and a numerical integration, which uses 1047.390 for the reciprocal mass of Jupiter and which has been fitted to the entire observational data set, are given in seconds of arc.

were those which had the largest minimum distance from Jupiter. Figure 4 compares the unfitted orbits with those which resulted from the six-unknown fit over the entire period of observations for the comets P/Grigg-Skjellerup, P/Kopff and P/PonsWinnecke. After adjusting these orbits with six-unknown solutions, a solution for seven unknowns was made. The resulting corrections to the assumed disturbing mass of Jupiter are listed in Table IV. As before, the correct answer should be -0.035 . Here the material has been arranged in order of increasing minimum separation from Jupiter.

TABLE IV

Solution for mass of Jupiter based on total data set

\begin{tabular}{lll}
\hline Comet & $\rho$ & $\Delta m$ \\
\hline P/Brooks 2 & 0.089 & -0.980 \\
P/Wolf & 0.119 & -0.887 \\
P/Oterma & 0.166 & -3.642 \\
P/Grigg-Skjellerup & 0.173 & -0.010 \\
P/Pons-Winnecke & 0.494 & -0.020 \\
P/Kopff & 0.577 & -0.047
\end{tabular}

$\rho=$ distance of minimum separation

\lrcorner $m=$ correction 10 reciprocal mass of Jupiter 


\section{Discussion}

Several important considerations can be deduced from the results as presented. From Table III, we see that the values for the correction to the mass deteriorate, not as a function of minimum distance to Jupiter but as a function of time elapsed from close approach. After a considerable time has elapsed from close approach, then distance to Jupiter plays a significant role.

From Figure 3 we see that an orbit based on an incorrect value for the mass of Jupiter could be forced to fit adequately the standard observations, provided we only used data up to the time around close approach in our fitting process. From our attempt to fit the standard artificial observations over the entire period they covered, we see that the ability to fit was a function of minimum separation at the time of close approach of the comet to Jupiter.

We can now draw the general conclusion that the six-unknown orbit-correction procedure used in this paper can be used to fit an orbit with an arbitrary mass of Jupiter to observations of comets which undergo a close approach to Jupiter if we do not try to include data far beyond the time of close approach. Furthermore, if we restrict ourselves to using observations not beyond 100 days after close approach, then the seven-unknown solution does correctly determine a correction to the mass of Jupiter and the orbit is meaningful. If we try to adjust an orbit using observations which extend considerably beyond close approach, then depending on the character of the comet orbit we might get seemingly meaningful, but nevertheless incorrect results.

The term 'seemingly meaningful' was purposely chosen. If we were working with real comet observations which are nonuniformly distributed and are imperfectly made, then we might be satisfied that we had adequately represented the observations.

Since we do not know the true mass of Jupiter, we hope that the value we use in practice is a sufficiently accurate representation of the true one. But to what should we attribute our inability to represent adequately the motions of some comets? We see that an incorrect value can lead to seemingly meaningful results in some cases and not in others. Perhaps, in those cases where we attribute deficiencies in our ability to represent the motion of comets to nongravitational forces, we might be able more correctly to attribute the deficiencies to inadequacies of our modeling. With respect to Figure 4, an important observation is to be made. It should be pointed out that the effect of an error in the mass of Jupiter occurs about one-half a revolution after a close approach or at perihelion passage of the comet.

From Figure 2 we can see that small changes in the mass of Jupiter can cause significant perturbations in the motions of those comets which come close to Jupiter. It seems that comets which have a close-approach distance less than 0.17 AU should be extremely sensitive indicators to an error in the mass of Jupiter. On further consideration, it is also possible to conclude that the observational history of such a comet does not have to be too long to get meaningful results. Of the objects studied in this investigation, P/Oterma had the most recent approach to Jupiter. This occurred in 1963 
(Marsden, 1970); unfortunately, this comet is now extremely faint. Despite this fact, it appears that a careful analysis of existing observations of this comet could give a reliable determination of the mass of Jupiter.

It was not possible to compare the results of this investigation, which used EckertBrouwer partial derivatives for the coefficients of six unknowns, with results obtained by using partial derivatives evaluated by numerically integrating the first-order variational equations. It is hoped that this study can be done sometime in the future.

\section{References}

Backlund, O.: 1894, Bull. Astron. 11, 473.

Brouwer, D. and Clemence, G. M.: 1961, Methods of Celestial Mechanics, Academic Press, New York and London.

Eckert, W. J. and Brouwer, D.: 1937, Astron. J. 46, 125.

Haerdtl, E.: 1889a, Denk. Acad. Wiss. Wien Math.-Nat. Kl. 56, 151.

Haerdtl, E.: 1889b, Astron. Nachr. 120, 257.

Herget, P.: 1970, private communication.

Makover, S. G.: 1956, Byull. Inst. Teor. Astron. 6, 67.

Makover, S. G. and Bokhan, N. A.: 1961, Trudy Inst. Teor. Astron. 8, 135.

Marsden, B. G.: 1968, Astron. J. 73, 367.

Marsden, B. G.: 1969, Astron. J. 74, 720.

Marsden, B. G.: 1970, Astron. J. 75, 75.

Marsden, B. G.: 1972, this Symposium, p. 239.

Möller, A.: 1872, Viertelj. Astron. Ges. 7, 85.

Porter, J. G.: 1961, Mem. Br. Astron. Assoc. 39, No. 3.

Rasmusen, H. Q.: 1967, Publ. Mind. Medd. Kbh. Obs. No. 194.

Roemer, E.: 1961, Astron. J. 66, 368.

Schubart, J. and Stumpff, P.: 1966, Veroeffentl. Astron. Rechen-Inst. Heidelberg No. 18.

\section{Discussion}

E. I. Kazimirchak-Polonskaya: Since you started with very approximate elements and integrated with a half-day step for $60 \mathrm{yr}$ the accumulation of error should be considerable.

W. J. Klepczynski: I performed integration at two-day intervals, one-day intervals, half-day intervals, and quarter-day intervals, and after $60 \mathrm{yr}$ of integration the coordinates all agree. But I should emphasize that I was merely trying to test the feasibility of the method, not make actual determinations of planetary masses, and for that reason it was not necessary for the elements to agree exactly with those of the real comets. 